\title{
Atomic Dynamics in Metallic Liquids and Glasses
}

\author{
T. Egami ${ }^{1,2,3}$, V. Levashov ${ }^{2}$, R. S. Aga ${ }^{3}$ and J. R. Morris ${ }^{1,3}$ \\ ${ }^{1}$ Department of Materials Science and Engineering, University of Tennessee, Knoxville, TN, USA \\ ${ }^{2}$ Department of Physics and Astronomy, University of Tennessee, Knoxville, TN, USA \\ ${ }^{3}$ Oak Ridge National Laboratory, Oak Ridge, TN, USA
}

How atoms move in metallic glasses and liquids is an important question in discussing atomic transport, glass formation, structural relaxation and other properties of metallic glasses. While the concept of free-volume has long been used in describing atomic transport, computer simulations and isotope measurements have shown that atomic transport occurs by a much more collective process than assumed in the free-volume theory. We introduce a new approach to describe the atomic dynamics in metallic glasses, in terms of local energy landscapes related to fluctuations in the topology of atomic connectivity. This approach may form the basis for a new paradigm for discussing the structureproperties relationship in metallic glasses. [doi:10.2320/matertrans.MJ200763]

(Received November 30, 2006; Accepted April 11, 2007; Published June 25, 2007)

Keywords: Atomic dynamics, metallic glasses, glass transition

\section{Introduction}

It is a challenging task to describe the atomic structure of liquids and glasses with sufficient clarity and accuracy, since the usual diffraction techniques provide only one-dimensional information on the structure in terms of the pairdensity function. But it is more daunting a task to relate the structure to the properties of metallic glasses, even if the structure were to be known. In the absence of a reliable framework to describe the structure-property relationship a century old concept of free-volume ${ }^{1)}$ is still widely used in this field. Indeed the free-volume theory by Cohen and Turnbull $^{2-4)}$ has successfully described why small changes in the volume can result in diffusivity changes which are very large, by more than ten orders of magnitude. However, from the onset they noted that the theory is less successful for metals. $^{2)}$ The critical free-volume, $v^{*}$, is only about $10 \%$ of the atomic volume, while it has to be almost as large as the atomic volume for the theory to be successful. Recent experiments and simulations further corroborate on this point. The pressure effect on diffusivity of metallic glasses ${ }^{5)}$ shows that the space between atoms involved in diffusion is much smaller than expected for the free-volume model; the activation volume for diffusion is much smaller than the atomic volume. Also the isotope effects on diffusion ${ }^{6)}$ fairly convincingly show that the picture of single atom diffusion is incorrect, and diffusion in liquids and glasses is a more collective phenomenon. Computer simulations ${ }^{7,8)}$ have captured the shape of the collective process, and showed that it is a chain-like process involving more than ten atoms, with each atom moving only slightly. These new results challenge us to rethink the mechanism of atomic transport in metallic glasses.

In this paper we propose a new framework for the description of atomic dynamics in metallic liquids and glasses, based upon the local fluctuations in the topology of atomic connectivity. We show that there are two energy scales in the energy landscape ${ }^{9)}$ that characterize the local topological fluctuations; the atomic bond energy $\left(V_{0}\right)$, and the local elastic deformation energy to change the local topology
$(W)$. The large energy scale, $V_{0}$, governs the high temperature atomic transport, and defines the crossover from pure liquid to viscous liquid. The smaller energy scale, $W$, determines the kinetics in the supercooled liquid, and thus the glass transition and the liquid fragility. At low temperatures topological fluctuations are spatially correlated, and bond breaking is accompanied by formation of a new bond, resulting in the bond-exchange. The bond-exchange results in diffusion and/or local shear deformation, depending on the geometry.

\section{Dynamics of Atomic Bonds in Metallic Systems}

The concept of atomic bond in glasses is usually used exclusively for covalent bonds, ${ }^{10)}$ and it is thought that bonds are not well defined in metallic glasses. However, in a dense random-packed structure ${ }^{11,12)}$ an atom is tightly surrounded by the nearest neighbour atoms. This local tight-packing results in the exclusion of second neighbour atoms from the shell of the nearest neighbour atoms. For this reason the first and second neighbours are fairly well separated. In addition, a pair-wise interatomic potential usually has a negative curvature at a distance between the nearest and second nearest neighbours, and this tends to separate the first and second nearest neighbours. ${ }^{2}$ Thus it is possible to define the atomic connectivity even in the metallic systems by the first peak of the pair-density function (PDF). For the sake of convenience we call the connectivity between atoms thus defined as "bond", without implications of electronic, directional bonds.

In a liquid metal these atomic bonds are cut and formed at a high rate. The distribution of the bond lifetimes determined by a molecular dynamics (MD) simulation using a modified Johnson potential for $\mathrm{Fe}^{13)}$ is shown in Fig. 1. ${ }^{14)}$ In this simulation the average nearest neighbour distance is $0.261 \mathrm{~nm}$, and the nearest neighbour was defined by the minimum in the PDF at $0.33 \mathrm{~nm}$ as the cut-off distance. Further details of the MD simulation will be presented elsewhere. ${ }^{14)}$ Two sub-peaks are seen in Fig. 1. The highfrequency sub-peak is due to vibrational motion of a pair of 


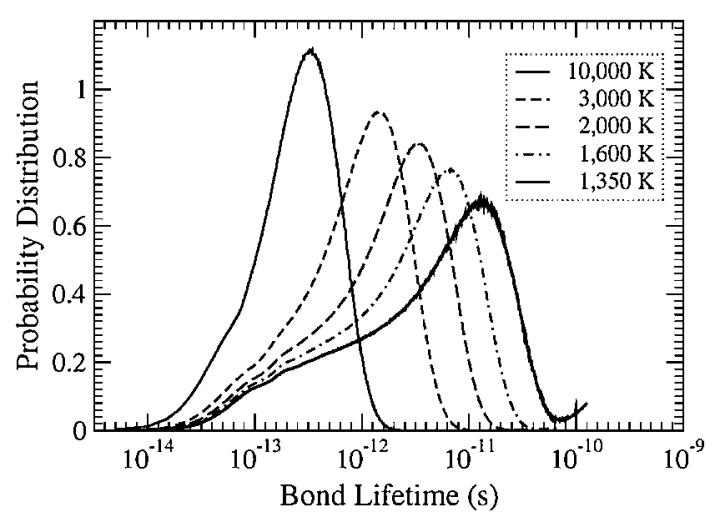

Fig. 1 Distribution of the bond lifetime in liquid Fe calculated by the MD simulation. ${ }^{14)}$

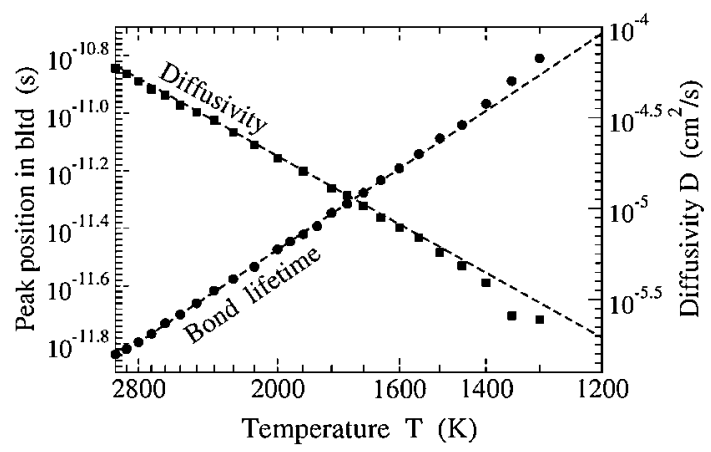

Fig. 2 Temperature dependence of the bond lifetime (circles, left axis) and diffusion coefficient (squares, right axis). ${ }^{14)}$

atoms with the separation close to the cut-off distance. The low-frequency sub-peak reflects the bond lifetime for the configurational changes, $\tau_{B}$, and its position shifts with temperature. The temperature dependence of the peak position is shown in Fig. $2{ }^{14)}$ and is compared to the diffusivity, $D$, determined by plotting $\langle r(t)\rangle^{2}$ against $t$, where $r(t)$ is the atomic displacement after time $t$. Both $D$ and $\tau_{B}$ show thermally activated behaviour, with the activation energy of $0.44 \mathrm{eV}\left(=1.75 V_{B}\right)$ for $\tau_{B}$ and $0.59 \mathrm{eV}\left(=2.34 V_{B}\right)$ for $D$, where $V_{B}(=0.25 \mathrm{eV})$ is the depth of the Johnson potential. The similarity in the behaviour of $D$ and $\tau_{B}$ suggests that diffusion at high temperatures takes place by collective bond diffusion. Because the activation energy is about twice the bond energy $V_{B}$, two bonds, not just one, are involved in bond diffusion. As shown in Fig. $3^{15)}$ if an atom cuts a bond and forms a new bond in the other side the atom will move by a small amount. Because of the threedimensionality an atom has to cut at least two bonds in order to move.

Such a bond-exchange can also cause shear deformation. If the new bond formed is perpendicular to the bond cut, the local structure becomes deformed by shear (Fig. 4). Indeed such bond-exchange was directly observed by x-ray diffraction for a metallic glass deformed by creep. ${ }^{16)}$ Thus parallel bond-exchange results in diffusion and perpendicular bondexchange produces shear deformation. In reality, of course, these two are mixed, and the statistical average determines the average response to the driving force. If there is only a
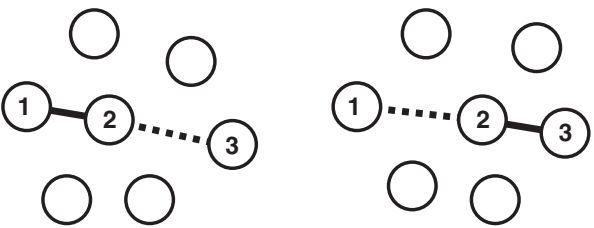

Fig. 3 Bond exchange process that leads to diffusion. ${ }^{15)}$ As the bond 1-2 (left) is cut and a new bond 2-3 is formed (right) the atom 2 moves to the right by a fraction of atomic distance.

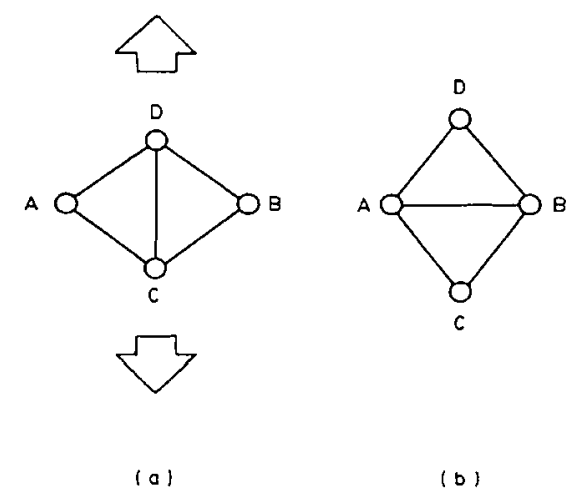

Fig. 4 Bond exchange that leads to shear deformation. ${ }^{16)}$ A tensile stress cuts the bond $\mathrm{C}-\mathrm{D}$, and a new bond $\mathrm{A}-\mathrm{B}$ is formed.

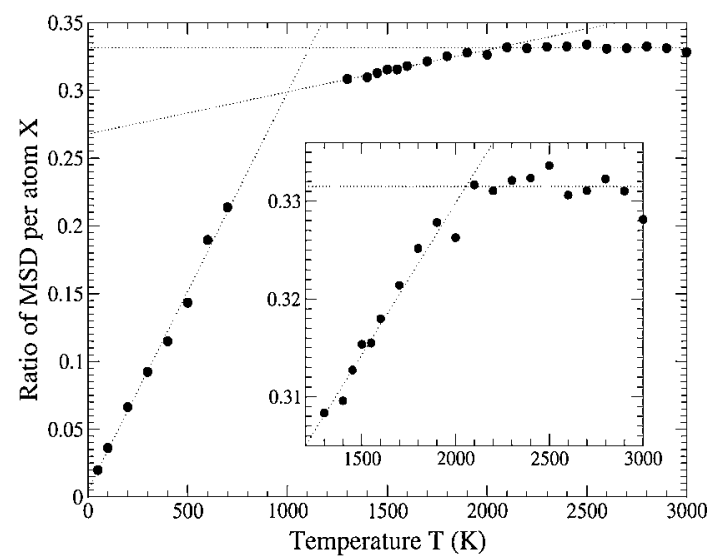

Fig. 5 Temperature dependence of the ratio of the fluctuation in the total number of bonds per atom, $\sigma_{B}{ }^{2}$, against the averaged distribution of the number of bonds at each atom $\left\langle\Delta N_{C}{ }^{2}\right\rangle{ }^{14)}$

density gradient local shear components will cancel each other resulting in no total shear. On the other hand if there is only a shear stress applied the diffusive motions will cancel out without producing atomic transport.

Figure 5 shows the temperature dependence of the ratio of the fluctuation in the total number of bonds per atom, $\sigma_{B}{ }^{2}$, against the averaged distribution of the number of bonds at each atom $\left\langle\Delta N_{C}{ }^{2}\right\rangle{ }^{14)}$ That $\sigma_{B}{ }^{2}$ being much smaller than $\left\langle\Delta N_{C}{ }^{2}\right\rangle$ suggests that the bond fluctuations are not independent, but are correlated. Furthermore, the ratio is nearly constant above $T^{*}=2000 \mathrm{~K}$, but decreases with decreasing temperature below. This crossover temperature is much above the glass transition temperature of the system, $T_{g}$, which is estimated to be around $800 \mathrm{~K} .{ }^{17)}$ It is interesting to note that this temperature $\left(k T^{*}=0.17 \mathrm{eV}\right)$ is close in energy 


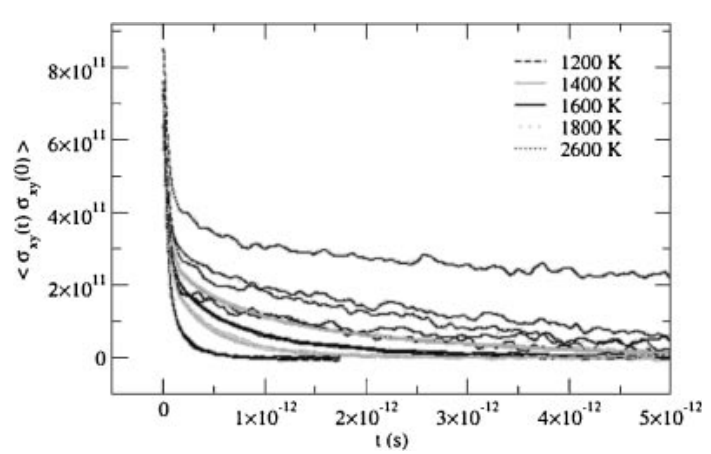

Fig. 6 Shear stress autocorrelation function, eq. (1), as a function of temperature. Above $1300 \mathrm{~K}$ all five shear stress correlation functions are the same, but at $1200 \mathrm{~K}$ (five dotted lines) they become different and irreproducible, due to the loss of ergodicity.

to $V_{B}$. It is possible that this temperature marks the trapping of the system to one of the mega-basins of the energy landscape. ${ }^{9)}$ Indeed the energy of the inherent structure calculated for this system shows a crossover around $2000 \mathrm{~K}$. Thus the activation energy for bond and atom diffusion, $2 V_{B}$, corresponds to the larger energy scale of the energy landscape, $V_{0}$.

On the other hand we found that the shear stress autocorrelation functions,

$$
\Gamma(t)=\frac{1}{N} \sum_{i}\left\langle\sigma_{i}^{x y}(0) \sigma_{i}^{x y}(t)\right\rangle,
$$

etc., become anisotropic below $T_{v}=1300 \mathrm{~K}$ as shown in Fig. 6. This means that the ergodicity is lost below this temperature. The mode-coupling temperature calculated from the positional autocorrelation function also shows some freezing around this temperature. The loss of ergodicity observed here must be the harbinger for the glass transition which is controlled by the smaller energy scale, $W$.

\section{Glass Transition}

As a supercooled liquid is further cooled its viscosity increases very rapidly, and at low enough temperatures it is so viscous that it appears like a solid. The glass transition temperature, $T_{g}$, is defined as a temperature where the viscosity reaches $10^{13}$ poise. How the viscosity of the supercooled liquid changes down to $T_{g}$ is best illustrated by the so-called Angell plot. ${ }^{18)}$ In this plot $\log \eta$, where $\eta$ is viscosity, is plotted against $T_{g} / T$. The slope just above $T_{g}$,

$$
m=\frac{\partial \log \eta}{\partial\left(T_{g} / T\right)}
$$

is called the fragility coefficient, and depends on composition. Liquids with large values of $m(m>25-30)$ are called fragile liquids, while those with small values of $m$ are called strong liquids. For fragile liquids the Angell plot changes the slope rather sharply around a temperature, $T_{s}$, forming a "knee" where the curvature reaches a maximum. Between $T_{g}$ and $T_{s}$, the temperature of the knee in the Angell plot, the viscosity changes about 13 orders of magnitude. Then the fragility coefficient, $m$, is given by,

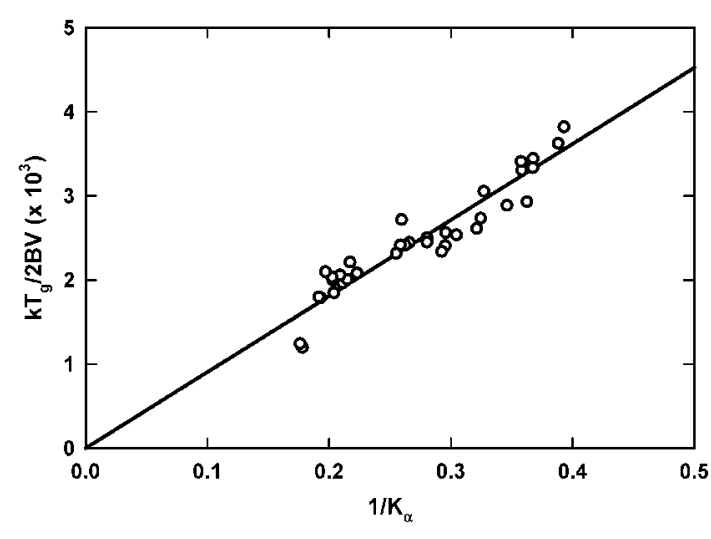

Fig. 7 Plot of $k T_{g} / 2 B V$ against $1 / K_{\alpha}$ for various metallic glasses, where $T_{g}$ is the glass transition temperature, $B$ bulk modulus, $\Omega$ atomic volume, and $K_{\alpha}$ is given by eq. (4). ${ }^{19)}$

$$
m \approx \frac{13}{1-\frac{T_{g}}{T_{s}}} .
$$

Since $m$ varies from one composition to another the mechanism to determine $T_{s}$ must be different from that for $T_{g}$.

It was found that $T_{g}$ is accurately described by

$$
\begin{aligned}
k T_{g} & =4 E_{v} \\
E_{V} & =\frac{B V}{2 K_{\alpha}}\left(\varepsilon_{v, T}\right)^{2}, \quad K_{\alpha}=\frac{3(1-v)}{2(1-2 \nu)}
\end{aligned}
$$

where $B$ is the bulk modulus, $V$ is the average atomic volume, $v$ is the Poisson's ratio, and $\varepsilon_{v, T}=0.095$ is the r.m.s. transformation volume strain at $T_{g}$, as shown in Fig. 7 that plots $k T_{g} / 2 B V$ against $1 / K_{\alpha} \cdot{ }^{19)}$ For our model of amorphous Fe eq. (4) gives $T_{g}=854 \mathrm{~K}$, in good agreement with the simulation. ${ }^{17)}$ However, in this case the agreement is totally fortuitous, since the simulation was done with the unrealistic cooling rate of $10^{13} \mathrm{~K} / \mathrm{s}$. A high cooling rate makes the liquid appear frozen at a viscosity much lower than $10^{13}$ poise. For an MD time of $\tau=10^{-8} \mathrm{~s}$. typically used in the present simulation, the Maxwellian relaxation time $\tau=\eta / G$, where $G$ is the shear modulus, gives $\eta=10^{4}$ poise for $G=10^{12}$ $\mathrm{erg} / \mathrm{cm}^{3}$, much smaller than $10^{13}$ poise. On the other hand a high cooling rate does not allow the liquid to undergo structural relaxation and keeps $\eta$ artificially low. These two errors partly cancel each other, and make the simulated value of $T_{g}$ not too far from reality. However, the effect is nothing but fortuitous, and usually the value of $T_{g}$ obtained by MD simulation cannot be taken seriously.

The derivation of eq. (4) requires a lengthy discussion. The energy of local density fluctuation, $E_{v}$, is given by

$$
E_{V}=\frac{B V}{2}\left(\varepsilon_{v, T}\right)^{2} \text {. }
$$

where $\varepsilon_{v, T}$ is the volume strain. In the glassy state, however, the local density fluctuation creates a long-range stress field which can be described by the Eshelby theory, ${ }^{20,21)}$ so that the elastic energy including the Eshelby relaxation effect is,

$$
E_{V}^{t}=\frac{B V}{2 K_{\alpha}}\left(\varepsilon_{v, T}\right)^{2}, \quad K_{\alpha}=\frac{3(1-v)}{2(1-2 v)},
$$

Now, if one places an atom $A$ with the radius of $r_{A}$ in the 


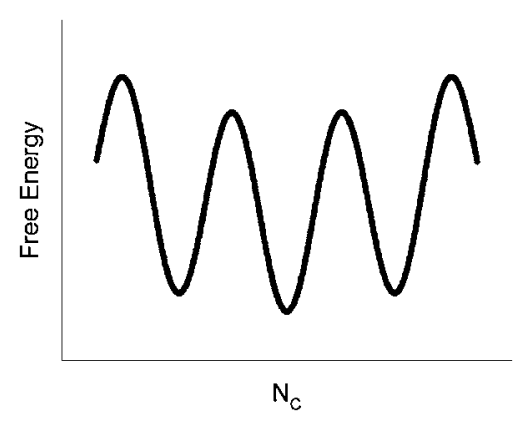

Fig. 8 Schematic diagram of the local energy landscape as a function of the local coordination number, $N_{C}$, where minima correspond to integral values of $N_{C} \cdot{ }^{23)}$

liquid of $B$ with the radius of $r_{B}$, the average coordination number of the atom $A, N_{C}(x)$, depends on the radius ratio, $x=r_{A} / r_{B}$. It can be heuristically shown ${ }^{22)}$ that,

$$
N_{C}(x)=4 \pi\left(1-\frac{\sqrt{3}}{2}\right)(1+x)(1+x+\sqrt{x(x+2)})
$$

While $N_{C}(x)$ given by eq. (7) is a continuous function of $x$, the actual coordination number is an integer. Thus eq. (7) is satisfied only when $N_{C}(x)=n$, where $n$ is an integer, at a value of $x, x_{n}=N_{C}^{-1}(n)$. In-between the structure is frustrated, and the network is locally distorted. If $x>x_{n}$, the central atom is under compression because the actual coordination is smaller than ideal. If $x<x_{n}$ it will be under negative (dilatational) pressure. This misfit pressure results in the local elastic energy, and this increases the local potential energy. Thus the local energy landscape must be an oscillating function with minima at $x=x_{n}$ and maxima inbetween (Fig. $8^{23)}$ ). The critical value of the change in $x$ corresponds to the half point between $x_{n}$ 's,

$$
\Delta x_{\text {crit }}=\frac{1}{2} / \frac{\partial N_{C}(x)}{\partial x} \text {, }
$$

and from eq. (7),

$$
\begin{aligned}
& \frac{\partial N_{C}(x)}{\partial x} \\
& \quad=8 \pi\left(1-\frac{\sqrt{3}}{2}\right)\left[1+x+\sqrt{x(x+2)}+\frac{1}{2 \sqrt{x(x+2)}}\right] .
\end{aligned}
$$

For $x=1$, it gives $\Delta x_{\text {crit }}=0.0369$, and the critical local volume strain,

$$
\varepsilon_{v}^{c r i t}(L)=\left.3 \Delta x\right|_{x=1}=\frac{6 \sqrt{3}-9}{4 \pi}=0.111 .
$$

This condition applied to uniform expansion was the basis for the compositional limit for glass formation in binary systems, ${ }^{24)}$

$$
c_{B}^{\min }=0.1 \frac{V}{|\Delta V|}
$$

which agrees well with experiments. Equation (10) defines the liquid-like sites as the sites with the local volume strain larger than 0.11 or smaller than -0.11 . The energy barrier corresponding to this critical strain,

$$
E_{V}^{c}=\frac{B V}{2 K_{\alpha}}\left(\varepsilon_{v}^{c r i t}\right)^{2}=6.16 \times 10^{-3} \frac{B V}{K_{\alpha}}
$$

gives the smaller energy scale, the depth of the local energy minima, $W$, in the energy landscape. In our model it is equal to $25 \mathrm{meV}$.

The local volume strain can be calculated from the local pressure, $\varepsilon=p / B$. The atomic-level pressure has a Gaussian distribution, ${ }^{20)}$ and its width of fluctuation follows a simple law at high temperatures, ${ }^{17)}$

$$
E_{V}=\frac{V\left\langle p^{2}\right\rangle}{2 B}=\frac{k T}{4},
$$

so that the distribution is given by

$$
P(p)=\frac{1}{\sqrt{2 \pi B k T / V}} \exp \left(-\frac{V p^{2}}{B k T}\right)
$$

Equation (13) means that the elastic energy of local deformation due to six components of the local stresses ${ }^{25}$ ) follows the equal partition law. ${ }^{17)}$ Equation (13), however, extrapolates to $p=0$ at $T=0$, while such a state is impossible to achieve in the random packed structure. This frustration is the root cause of the glass transition. Since the local pressure, thus the local volume, has a Gaussian distribution given by (14), the total density of the liquidlike sites is given by,

$$
p(l i q)=C E\left(y_{C}\right)=\frac{2}{\sqrt{\pi}} \int_{y_{C}}^{\infty} e^{-y^{2}} d y
$$

where

$$
y_{C}=\frac{\varepsilon_{v}^{c r i t}(L)}{\sqrt{2}\left\langle\varepsilon_{v}^{2}\right\rangle^{1 / 2}} .
$$

For $\varepsilon_{v}=0.095$ it gives

$$
p(l i q)=C E\left(y^{c r i t}\right)=0.243,
$$

which is close to the percolation concentration for the dense random packed structure, 0.2. ${ }^{26)}$ Thus eq. (4) explains the glass transition in terms of the percolation of the liquid-like sites as envisaged by Cohen and Grest. ${ }^{27)}$

In the liquid state the energy of the volume fluctuation is given by eq. (5), without the elastic relaxation factor of $K_{\alpha}$. Following the same argument that led to eq. (4) but without the factor $K_{\alpha}$, a transition temperature $T_{T}$ can be defined;

$$
k T_{T}=2 B V\left(\varepsilon_{v, T}\right)^{2} .
$$

This gives $T_{T}=2260 \mathrm{~K}$ for our model, which agrees with the crossover temperature shown in Fig. 5. In the high-temperature liquid state the atomic bond lifetime is very short as shown in Fig. 1. Thus there is no time for the atomic environment to relax in the Eshelby fashion to produce the long-range stress field. The density of the sites with the instability condition defined for such short lifetimes, reaches the percolation concentration at $T_{T}$. Thus this crossover may be caused by the dynamic instability of the nearest neighbour cage in the liquid state.

\section{Mechanical Deformation}

When a shear stress is applied the local topology around an atom becomes unstable at a certain critical shear strain, just as the same way as the criticality of the volume strain, eq. (10). This value of shear instability has not been 
calculated yet, but the following heuristic argument gives some clue. The atomic-level stress is a $3 \times 3$ symmetric tensor $^{25)}$ that can be grouped into an isostatic stress, pressure ( $\ell=0$ in the spherical representation), and five shear stress components $(\ell=2) .^{20)}$ At high temperatures the shear stresses also follow the same temperature dependence as eq. (13);

$$
E_{v}=\frac{V\left\langle\tau^{2}\right\rangle}{10 G}=\frac{k T}{4}
$$

where $\tau^{2}$ is the sum of the squares of each components. Thus we can define a shear freezing temperature by

$$
k T_{s}=2 G V\left(\varepsilon_{s}^{c}\right)^{2}
$$

The nature of this transition is unclear, but we assume it is the cause of the knee in the Angell plot. In the limit of $\mathrm{G} \rightarrow 0$, or $v \rightarrow 0.5, T_{s}$ has to be equal to $T_{g}$, since shear resistance is absent and both are controlled by bulk modulus. This yields

$$
\frac{\left\langle\left(\varepsilon_{s}^{c}\right)^{2}\right\rangle}{\left\langle\left(\varepsilon_{v}^{c}\right)^{2}\right\rangle}=\frac{\langle B\rangle}{\langle G\rangle K_{\alpha}}=\frac{1+v}{1-v}=3,
$$

and

$$
\frac{T_{s}}{T_{g}}=\frac{3(1-v)}{1+v} .
$$

Thus,

$$
\varepsilon_{s}^{c r i t}=\sqrt{3} \varepsilon_{v}^{T, c r i t}=0.192 .
$$

From eqs. (3) and (22),

$$
m=\frac{39(1-v)}{2(1-2 v)}=13 K_{\alpha}
$$

which shows good agreement. ${ }^{28)}$

By observing the metallic glasses anelastically deformed by creep with $\mathrm{x}$-ray diffraction, the microscopic mechanism of shear deformation was found to be the perpendicular bondexchange as shown in Fig. 4. ${ }^{17)}$ The critical shear strain for such deformation has not been calculated, but the estimate by eq. (23) is reasonable. The task of connecting the critical shear strain to the macroscopic yield point remains to be carried out. It is important to note that the local shear modulus $G$ has a continuous distribution down to zero. ${ }^{20)}$ This means that always some atoms are ready to yield plastically for infinitesimal strain. The average shear modulus of a glass is lowered by such atomic rearrangement by as much as $25 \% .^{29)}$ If we calculate the shear modulus by actually deforming the model in the MD simulation the value of $G$ is that much lower than the value calculated for homogeneous strain. Since the number of atomic rearrangement is proportional to the strain the response of a glass appears elastic, but it is intrinsically anelastic, because always some bonds are rearranged no matter how small the strain is. ${ }^{30)}$ In other words even well below $T_{g}$ there are always liquid-like sites, and they respond anelastically to the stress. The two-level tunneling states ${ }^{31)}$ must be just these liquid-like states.

\section{Conclusions}

Creating an atomistic theory of glasses is a very challeng- ing task, since just characterizing the structure itself is already a daunting task. For metallic glasses the job may be slightly easier, since the unit is an atom with a basically spherical potential. Unlike covalent oxide glasses bonding rules, thus chemistry, interferes less. The main principle is the packing efficiency, and if the right language is found the local geometry and energetics can be formulated. We believe that the right language is the topological fluctuations of the atom connectivity network expressed in terms of the atomiclevel stresses. While the theory of topological fluctuations is still in the stage of development, already initial results are quite encouraging. It is hoped that this will lead to a more general theory of glasses and liquids.

\section{Acknowledgments}

The authors are grateful to J. C. Phillips, M. F. Thorpe, C. A. Angell, A. L. Greer, S. J. Poon, V. N. Novikov and C. T. Liu for useful discussions. This work was supported by the Division of Materials Science and Engineering, Office of Basic Energy Sciences (LLH), U.S. Department of Energy under contract DE-AC05-00OR-22725 with UT-Battele, LLC.

\section{REFERENCES}

1) A. J. Batschinski: Z. Physic. Chem. 84 (1913) 643-706.

2) M. H. Cohen and D. Turnbull: J. Chem. Phys. 31 (1959) 1164-1169.

3) D. Turnbull and M. H. Cohen: J. Chem. Phys. 34 (1961) 120-125.

4) D. Turnbull and M. H. Cohen: J. Chem. Phys. 52 (1970) 3038-3041.

5) P. Klugkist et al.: Phys. Rev. Lett. 80 (1998) 3288-3291.

6) F. Faupel, W. Frank, M.-P. Macht, H. Mehrer, V. Naundorf, K. Rätzke, H. R. Schober, S. K. Sharma and H. Teichler: Rev. Mod. Phys. 75 (2003) 237-280

7) H. R. Schober: Physica A 201 (1993) 14-24.

8) C. Donati, J. F. Douglas, W. Kob, S. J. Plimpton, P. H. Poole and S. C. Glotzer: Phys. Rev. Lett. 80 (1998) 2338-2341.

9) D. J. Wales: Energy Landscapes (Cambridge University Press, Cambridge, 2003).

10) J. C. Phillips: J. Non-Cryst. Solids 34 (1979) 153-181.

11) J. D. Bernal and J. Mason: Nature (London) 188 (1960) 910-911.

12) G. D. Scott: Nature (London) 188 (1960) 908-910.

13) T. Tomida and T. Egami: Phys. Rev. B 52 (1995) 3290-3308.

14) V. Levashov, T. Egami, R. S. Aga and J. R. Morris, unpublished.

15) T. Egami: Rep. Prog. Phys. 47 (1984) 1601-1725.

16) Y. Suzuki, J. Haimovic and T. Egami: Phys. Rev. B 35 (1987) 21622168.

17) S.-P. Chen, T. Egami and V. Vitek: Phys. Rev. B 37 (1988) 2440-2449.

18) C. A. Angell: Science 267 (1995) 1924-1935.

19) T. Egami, S. J. Poon, Z. Zhang and V. Keppens: Phys. Rev. B, in press.

20) T. Egami and D. Srolovitz: J. Phys. F: Metal Phys. 12 (1982) 2414 2463.

21) J. D. Eshelby: Proc. Roy. Soc. London A 241 (1957) 376-396.

22) T. Egami and S. Aur: J. Non-Cryst. Solids 89 (1987) 60-74.

23) T. Egami: Mater. Trans. 43 (2002) 510-517.

24) T. Egami and Y. Waseda: J. Non-Crsyt. Solids 64 (1984) 113-134.

25) T. Egami, K. Maeda and V. Vitek: Phil. Mag. A 41 (1980) 883-901.

26) M. J. Powell: Phys. Rev. B 21 (1980) 3725-3728.

27) M. H. Cohen and G. Grest: Phys. Rev. B 20 (1979) 1077-1098.

28) T. Egami and S. J. Poon: unpublished.

29) L. A. Davis: Mechanics of metallic glasses. Rapidly quenched metals, eds. Grant, N. J. and B. C. Giessen (MIT Press, Cambridge, 1976) p. 369-391.

30) Y. Suzuki and T. Egami: J. Non-Cryst. Solids 75 (1985) 361-366.

31) P. W. Anderson, B. I. Halperin and C. M. Varma: Phil. Mag. 25 (1972) $1-9$. 